\title{
INFLUÊNCIA DA FUNCIONALIZAÇÃO DA SUPERFÍCIE NAS PROPRIEDADES FÍSICO-QUÍMICAS DE CATALISADORES DE CARBETO DE TUNGSTÊNIO.
}

\author{
G. M. V. PINHEIRO ${ }^{1}$, D. M. MEIRA² e C. B. RODELLA ${ }^{2}$ \\ ${ }^{1}$ Universidade Estadual de Campinas, Faculdade de Engenharia Química \\ ${ }^{2}$ Laboratório Nacional de Luz Síncrotron - LNLS \\ E-mail para contato: cristiane.rodella@lnls.br
}

\begin{abstract}
RESUMO - As propriedades físico-químicas dos catalisadores estão intimamente relacionadas ao seu desempenho catalítico. O objetivo deste trabalho é o estudo da influência da funcionalização da superfície e da adição de metal em catalisadores de carbeto de tungstênio, suportados em carvão e aplicados à reação de hidrogenação do ácido levulínico à $\gamma$-valerolactona. Para tanto, houve a preparação dos suportes funcionalizados com bases de diferentes forças $(\mathrm{KOH}$, $\mathrm{K}_{2} \mathrm{CO}_{3}$ e $\mathrm{KHCO}_{3}$ ) e do catalisador promovidos com Ni. Até o presente momento, foi possível observar que o $\mathrm{KOH}$ promove uma maior dispersão das partículas de níquel e do carbeto de tungstênio. $\mathrm{O}$ níquel mais disperso permite que ambas as fases $\mathrm{W}_{2} \mathrm{C}$ e WC do carbeto de tungstênio estejam presentes no catalisador. As caracterizações estão sendo realizadas para as amostras funcionalizadas com $\mathrm{K}_{2} \mathrm{CO}_{3}$ e $\mathrm{KHCO}_{3}$. Ao final, a atividade catalítica será avaliada e espera-se determinar uma correlação catálise/estrutura do catalisador, e assim os fatores que impactam a atividade catalítica.
\end{abstract}

\section{INTRODUÇÃO}

A produção de produtos químicos com alto valor agregado e biocombustíveis a partir de fontes renováveis de energia é de extrema importância para suprir a demanda energética dos setores industriais e de transporte. A conversão catalítica da biomassa lignocelulósica e de seus derivados pode ser econômica e ambientalmente viáveis na obtenção de produtos bioderivados, como observado por Serrano-Ruiz et al (2012) e Climent et al (2014).

Carbetos de tungstênio têm se mostrado catalisadores eficientes na conversão de celulose e seus derivados em reações de hidrogenação, obtendo produtos de alto valor comercial. De acordo com trabalhos conduzidos por Alvarez-Merino et al (2000) e Ji et al (2008), o tamanho de partícula, a dispersão e a adição de promotores nesses catalisadores influencia o processo de carburação assim como a performance catalítica. No caso da conversão de biomassa também é importante considerar as propriedades ácido-base dos catalisadores uma vez que essas influenciam diretamente no mecanismo de reação e seletividade.

Embora catalisadores de carbeto de tungstênio promovidos com níquel tem sido explorados com sucesso na conversão de biomassa, a interação entre promotor-tungstênio e o processo de carburação ainda não foi explorado com profundidade. 
Assim, o objetivo desse trabalho é a preparação de catalisadores de carbeto de tungstênio suportados em carvão ativo e promovidos com níquel tendo sido a superfície funcionalizada com bases de diferentes forças: $\mathrm{KOH}, \mathrm{KHCO}_{3}$ e $\mathrm{K}_{2} \mathrm{CO}_{3}$. As propriedades estruturais do catalisador e seu desempenho na reação de conversão de ácido levulínico a $\gamma$ valerolactona serão avaliados em relação à adição de níquel e funcionalização da superfície.

\section{MATERIAIS E MÉTODOS}

A preparação dos catalisadores se deu em três etapas: (i) funcionalização do carvão ativo, (ii) impregnação com paratungstato de amônio, (iii) impregnação com níquel. Em seguida, foram realizados o (iv) tratamento térmico e a (v) caracterização dos catalisadores. Cada uma das etapas será detalhada a seguir.

\subsection{Funcionalização do Carvão Ativo}

A 4,0 g de carvão seco a $100^{\circ} \mathrm{C}$ por uma noite adicionou-se um volume de $120 \mathrm{~mL}$ de solução $0,4 \mathrm{~mol}$ de $\mathrm{KOH}$. A mistura de carvão e base foi agitada por aproximadamente 10 minutos a temperatura ambiente. Em seguida, elas foram centrifugadas a $3000 \mathrm{rpm}$ por 5 minutos e postas para secar em estufa. Subseqüentemente, as amostras foram tratadas a $300^{\circ} \mathrm{C}$ em fluxo de $\mathrm{N}_{2}$ por 1 hora. $\mathrm{O}$ mesmo procedimento foi repetido utilizando-se as bases $\mathrm{K}_{2} \mathrm{CO}_{3}$ e $\mathrm{KHCO}_{3}$.

Solução de $\mathrm{HCl} 25 \% \mathrm{~m} / \mathrm{m}$ foi utilizada para a lavagem da amostra, com o intuito de retirar as bases fisissorvidas. As equações correspondentes a cada caso são:

$\mathrm{HCl}_{(\mathrm{aq})}+\mathrm{KOH}_{(\mathrm{aq})} \rightarrow \mathrm{KCl}_{(\mathrm{aq})}+\mathrm{H}_{2} \mathrm{O}_{(\mathrm{aq})}$ (Carvão funcionalizado com $\mathrm{KOH}$ ) $2 \mathrm{HCl}_{(\mathrm{aq})}+\mathrm{CO}_{3}{ }^{2-}{ }_{(\mathrm{aq})} \rightarrow \mathrm{H}_{2} \mathrm{O}_{(\mathrm{l})}+\mathrm{CO}_{2(\mathrm{~g})}+2 \mathrm{Cl}_{(\mathrm{aq})}^{-}$(Carvão funcionalizado com $\mathrm{K}_{2} \mathrm{CO}_{3}$ ) $\mathrm{HCl}_{(\mathrm{aq})}+\mathrm{HCO}_{3_{(\mathrm{aq})}^{-}}^{-} \rightarrow \mathrm{H}_{2} \mathrm{O}_{(\mathrm{l})}+\mathrm{CO}_{2(\mathrm{~g})}+\mathrm{Cl}_{(\mathrm{aq})}^{-}$(Carvão funcionalizado com $\left.\mathrm{KHCO}_{3}\right)$

Monitorando-se com pHmetro, adicionou-se $\mathrm{HCl}$ à suspensão de carvão até o sobrenadante ter $\mathrm{pH}$ igual ao da água destilada. Então, centrifugou-se a mistura e adicionou-se o carvão em água novamente (formando uma suspensão). Enquanto o pH era superior ao da água destilada, repetia-se o procedimento. Quando os dois valores de $\mathrm{pH}$ enfim se igualaram, o carvão funcionalizado foi separado do sobrenadante (por meio da centrífuga) e seco na estufa $\left(100^{\circ} \mathrm{C}\right)$.

\subsection{Impregnação com Paratungstato de Amônio}

Carvão funcionalizado foi impregnado com 30\% de W. Para isso, utilizou-se o método de impregnação incipiente de uma solução de paratungstato de amônio $\left(\left(\mathrm{NH}_{4}\right)_{10} \mathrm{~W}_{12} \mathrm{O}_{41} .5 \mathrm{H}_{2} \mathrm{O}\right)$. Brevemente, a cada 30 minutos, pipetavam-se 10 gotas de solução de paratungstato e adicionava-se ao carvão funcionalizado, homogeneizando-se com pistilo. No intervalo das adições o carvão era seco em estufa a $100^{\circ} \mathrm{C}$. Repetiu-se o procedimento até o fim da solução de paratungstato, a qual, durante o processo, foi mantida a $75^{\circ} \mathrm{C}$ para garantir que o sal estivesse completamente solubilizado. Ao final, a amostra de carvão impregnado descansou por uma noite $\left(100^{\circ} \mathrm{C}\right)$. 


\subsection{Impregnação com Níquel}

As amostras impregnadas com tungstênio foram então impregnadas com $2 \% \mathrm{Ni}$. Nitrato de níquel $\left(\mathrm{Ni}\left(\mathrm{NO}_{3}\right)_{2}\right)$ foi utilizado como sal precursor de níquel e mais uma vez o procedimento realizado foi o método da impregnação incipiente. Ao final, as diferentes amostras de carvão impregnado descansaram em estufa por uma noite $\left(100^{\circ} \mathrm{C}\right)$.

\subsection{Tratamento Térmico}

Após as impregnações, os catalisadores $(500 \mathrm{mg})$ passaram pelo tratamento térmico de carburação. Primeiramente as amostras foram secas a $400{ }^{0} \mathrm{C}\left(10{ }^{\circ} \mathrm{C} / \mathrm{min}\right)$ por $40 \mathrm{~min}$ sob fluxo de $\mathrm{He}(50 \mathrm{~mL} / \mathrm{min})$. Em seguida, foram aquecidas $\left(10{ }^{\circ} \mathrm{C} / \mathrm{min}\right.$, razão $\left.\mathrm{H}_{2} / \mathrm{CH}_{4} 90 / 10\right)$ a $780{ }^{\circ} \mathrm{C}$ e mantidas nessa temperatura por $40 \mathrm{~min}$, ocorrendo a carburação propriamente dita. $\mathrm{O}$ resfriamento até temperatura ambiente foi realizado somente sob o fluxo de $\mathrm{H}_{2}$. Em seguida, as amostras foram passivadas $\left(\mathrm{O}_{2} / \mathrm{He} 1 / 24\right)$ durante toda a noite. A passivação é feita no sentido de criar uma monocamada de óxido de tungstênio na superfície do catalisador. Ao final da preparação as amostras foram denominadas segundo a funcionalização com as bases e impregnação com Ni conforme descrito na Tabela 1.

Tabela 1 - Denominação das amostras conforme procedimento de preparação

\begin{tabular}{|c|c|c|c|}
\hline $\begin{array}{c}\text { Amostras funcionalizadas } \\
\text { com as bases }\end{array}$ & Denominação & $\begin{array}{c}\text { Amostras funcionalizadas } \\
\text { com as bases e } \\
\text { impreganadas com Ni }\end{array}$ & Denominação \\
\hline Sem funcionalização & $\mathrm{W}_{\mathrm{x}} \mathrm{C}$ & Sem funcionalização $+\mathrm{Ni}$ & $\mathrm{Ni} \mathrm{W}_{\mathrm{x}} \mathrm{C}$ \\
\hline $\mathrm{KOH}$ & $\mathrm{W}_{\mathrm{x}} \mathrm{C} \_\mathrm{OH}$ & $\mathrm{KOH}+\mathrm{Ni}$ & $\mathrm{Ni}$ - $\mathrm{W}_{\mathrm{x}} \mathrm{C} \mathrm{OH}$ \\
\hline $\mathrm{KHCO}_{3}$ & $\mathrm{~W}_{\mathrm{x}} \mathrm{C} \mathrm{HCO}_{3}$ & $\mathrm{KHCO}_{3}+\mathrm{Ni}$ & $\mathrm{Ni} \mathrm{W}_{\mathrm{x}} \mathrm{C} \mathrm{HCO}_{3}$ \\
\hline $\mathrm{K}_{2} \mathrm{CO}_{3}$ & $\mathrm{~W}_{\mathrm{x}} \mathrm{C} \mathrm{CO}_{3}$ & $\mathrm{~K}_{2} \mathrm{CO}_{3}+\mathrm{Ni}$ & $\mathrm{Ni} \mathrm{W}_{\mathrm{x}} \mathrm{C} \mathrm{CO}_{3}$ \\
\hline
\end{tabular}

\subsection{Caracterizações dos Catalisadores}

A análise de fisissorção de nitrogênio foi realizada para determinar a área superficial específica dos catalisadores (utilizando método BET). Primeiramente, as amostras foram secas a $120{ }^{\circ} \mathrm{C}$ por $16 \mathrm{~h}$. Em seguida, as isotermas de adsorção e dessorção de $\mathrm{N}_{2}\left(-196{ }^{\circ} \mathrm{C}\right)$ foram realizadas em um equipamento Autosorb 1C (Quantachrome) no Laboratório Nacional de Luz Síncrotron (LNLS).

As medidas de difração de raios X (DRX), dos catalisadores, foram realizadas na linha XPD do LNLS (energia $8 \mathrm{KeV}$ ). O intervalo $2 \theta$ medido foi 20 a $100^{\circ}$, passo de $0.050 \mathrm{~s}^{-1}$, utilizando-se o detector Mythen (distância amostra detector de $1 \mathrm{~m}$ ).

\section{RESULTADOS E DISCUSSÃO}

Até o presente momento a avaliação dos resultados foi realizada para as amostras: WxC, WxC_OH, Ni_WxC e Ni_WxC_OH e serão aqui apresentados. Os demais resultados ainda estão sendo avaliados e serão apresentados no congresso. 
Os resultados de área superficial específica são apresentados na Tabela 2.

Tabela 2 - Resultados de área superficial específica

\begin{tabular}{|c|c|}
\hline Amostras & Área $\left(\mathrm{m}^{2} / \mathrm{g}\right)$ \\
\hline $\mathrm{W}_{\mathrm{x}} \mathrm{C}$ & 544 \\
\hline $\mathrm{W}_{\mathrm{x}} \mathrm{C}-\mathrm{OH}$ & 491 \\
\hline $\mathrm{Ni} \mathrm{W}_{\mathrm{x}} \mathrm{C}$ & 581 \\
\hline $\mathrm{Ni} \mathrm{W}_{\mathrm{x}} \mathrm{C} \mathrm{OH}$ & 600 \\
\hline
\end{tabular}

Os resultados, de área superficial específica, obtidos parecem indicar que a adição do metal (Ni) está associada a uma maior área superficial específica do catalisador, promovendo uma maior dispersão do carbeto de tungstênio. De fato, Rodella et al. (2015) observaram que a impregnação de Ni em carbetos de tungstênio auxilia na obtenção de partículas pequenas e bem dispersas, o que acarreta em maior área superficial. $\mathrm{O}$ efeito dessa dispersão parece ser mais pronunciado se o carvão tiver sido funcionalizado com a base $\mathrm{KOH}$, uma vez que nessas amostras, os grupos básicos parecem aumentar a dispersão tanto das partículas de Ni como do carbeto de tungstênio.

Através das análises posteriores das áreas superficiais das amostras funcionalizadas com $\mathrm{KHCO}_{3}$ e $\mathrm{K}_{2} \mathrm{CO}_{3}$ será possível estabelecer uma correlação entre a força das bases e as propriedades texturais do catalisador.

Os difratogramas de raios X, após a carburação, são apresentados na Figura 1.

Figura 1 - Difratogramas de raios X após a carburação das amostras não funcionalizadas e funcionalizadas com $\mathrm{KOH}$. $\operatorname{Em}(\mathrm{A})$, o carvão foi impregnado com tungstênio e em (B), o carvão foi impregnado com tungstênio e níquel.
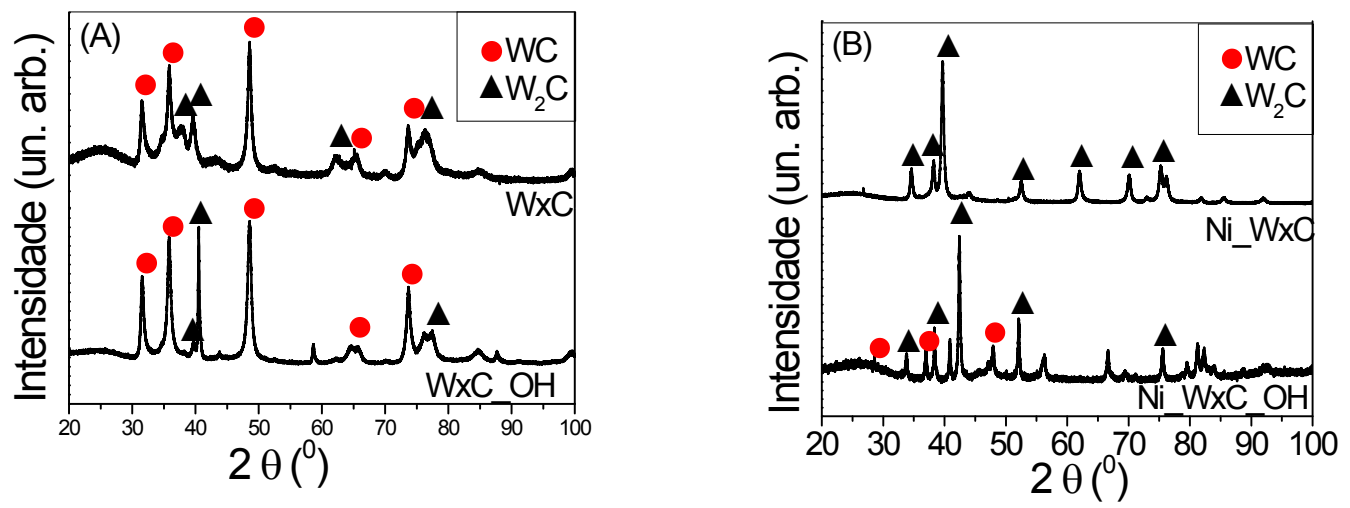

A análise dos difratogramas indica que as fases $\mathrm{WC} \mathrm{e} \mathrm{W}_{2} \mathrm{C}$ foram formadas durante a carburação. Na ausência de Ni (Figura 1A), as amostras encontram-se menos cristalinas (picos mais alargados) e com uma maior mistura entre essas duas fases. Por outro lado, a adição de Ni (Figura 1B) parece estar associada a uma maior cristalinidade (picos mais estreitos) das amostras e a predominância da fase $\mathrm{W}_{2} \mathrm{C}$. 
Como afirmado por Ji et al (2008), na etapa de carburação das amostras, o tungstênio passa pelas seguintes mudanças de fases:

$\mathrm{WO}_{3} \rightarrow \mathrm{W}_{\mathrm{X}} \mathrm{O}_{\mathrm{Y}} \rightarrow \mathrm{WO}_{2} \rightarrow \mathrm{W} \rightarrow \mathrm{W}_{2} \mathrm{C} \rightarrow \mathrm{WC}$

$\mathrm{W}_{\mathrm{X}} \mathrm{O}_{\mathrm{Y}}$ é uma fase não estequiométrica do tungstênio.

Tipicamente, a carburação se processa até a fase WC. Todavia, o Ni aparentemente estabiliza a fase metaestável $\mathrm{W}_{2} \mathrm{C}$. Rodella et al. (2015) concluiu que de fato o Ni auxiliava na estabilização da fase metaestável $\mathrm{W}_{2} \mathrm{C}$, porque ele induz a deposição de carbono polimérico (resultante do $\mathrm{CH}_{4}$ do fluxo, que sofre redução) na superfície do catalisador, o que impede uma posterior redução à fase $\mathrm{WC}$, parando a sequência de reações na fase metaestável $\mathrm{W}_{2} \mathrm{C}$. Leal (2014) observou também comportamento similar quando o paládio foi utilizado como promotor.

A Figura 1B mostra também que níquel altamente disperso, devido à presença do grupo básico proveniente do $\mathrm{KOH}$, pode influenciar no aparecimento de ambas as fases $\mathrm{W}_{2} \mathrm{C}$ e WC após a carburação, não privilegiando completamente a fase $\mathrm{W}_{2} \mathrm{C}$.

\section{CONCLUSÃO}

Até o presente momento, a funcionalização do carvão com $\mathrm{KOH}$ está relacionada a uma maior área superficial do catalisador. Isso parece indicar que uma maior dispersão do níquel e do carbeto de tungstênio. Essa maior dispersão, por sua vez, parece influenciar no aparecimento de ambas as fases $\mathrm{WC}_{\mathrm{e}} \mathrm{W}_{2} \mathrm{C}$ após a carburação, de acordo com o difratograma obtido. Será analisado como as bases $\mathrm{KHCO}_{3}$ e $\mathrm{K}_{2} \mathrm{CO}_{3}$, de menores forças, impactam na fase de carbeto de tungstênio observada. Além disso, um estudo posterior será realizado aonde as atividades catalíticas de cada um dos catalisadores sintetizados serão correlacionadas com a fase predominate do carbeto de tungstênio.

\section{AGRADECIMENTOS}

Agradecimentos ao CNPq-PIBIC pela Bolsa de Iniciação Científica concedida e ao Laboratório de Química do LNLS/CNPEM pela infraestrutura oferecida.

\section{REFERÊNCIAS BIBLIOGRÁFICAS}

ALVAREZ-MERINO, M. A.; CARRASCO-MARÍN, F.; FIERRO, J. L. G.; MORENOCASTILHA, C. J. Catal., v. 192, p. 363-373, 2000.

CLIMENT, M. J.; CORMA, A.; IBORRA, S. Green Chem., v. 16, p. 516-547, 2014.

GIRAUDON, J. M.; DEVASSINE, P.; LAMONIER, J. F.; DELANNOY, L.; LECLERQ, L.; LECLERCQ, L. J. Solid State Chem., v. 154, p. 412-426, 2000.

JI, N.; ZHANG, T. ET AL. Angew. Chem. Int. Ed 47 (2008) 8510.

LEAL, G. F., v. 5, p. 23874-23885, 2014.

RODELLA, C. B.; BARRETT, D. H.; MOYA, S. F.; FIQUEROA, S. J. A.; PIMENTA, M. T. B.; CURVELO, A. A. S.; SILVA; V. T. Physical and chemical studies of tungsten 
carbide catalysts: effects of Ni promotion and sulphonated carbon. RSD Advance. Ed. 30, 2015.

SERRANO-RUIZ, J. C.; PINEDA, A.; BALU, A.; LUQUE, R.; CAMPELO, J. M.; ROMERO, A. A.; RAMOS-FERNÁNDEZ, J. M.. Catal. Today, v. 195, p. 162-168, 2012. 